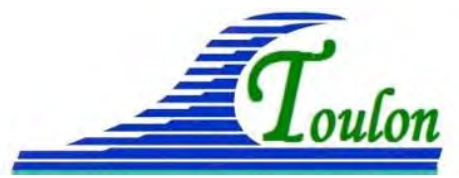

XIV $V^{\text {èmes }}$ Journées Nationales Génie Côtier - Génie Civil Toulon, 29 juin au $1^{\text {er juillet } 2016}$

DOI:10.5150/jngcgc.2016.026 $\quad$ (C) Editions Paralia CFL

disponible en ligne - http://www.paralia.fr - available online

\title{
Modélisation du potentiel de transport sédimentaire longitudinal des plages du Languedoc-Roussillon
}

\author{
Benjamin KULLING ${ }^{1}$, François SABATIER ${ }^{1}$, Edward ANTHONY ${ }^{1}$
}

1. Aix-Marseille Université, UM 34 CEREGE, BP80, 13545 Aix-en-Provence Cedex 04, France.

kulling@cerege.fr

\section{Résumé :}

Une approche modélisatrice du transport longitudinal le long des plages du LanguedocRoussillon est proposée sur la base du modèle numérique Unibest-LT (Uniform Beach Sediment Transport) en utilisant en entrée des données de houle de 1979 à 2010 (base de données ANEMOC-2) ainsi que des relevés topo-bathymétriques à haute résolution (LiDAR). Unibest-LT permet de calculer le Transport Sédimentaire Longitudinal annuel Résultant $\left(\mathrm{TSL}_{\mathrm{R}}\right)$ sur l'ensemble du linéaire côtier étudié. Les directions du TSL modélisé concordent bien avec celles définies empiriquement et nos travaux précisent et/ou dévoilent des dérives littorales potentielles unidirectionnelles ou bidirectionnelles selon les secteurs.

Mots-clés : Dérive littorale, Plages sableuses, Unibest-LT.

\section{Introduction}

En ingénierie côtière, le transit sédimentaire longitudinal dans la zone de surf est généralement appréhendé selon les travaux pionniers de LONGUET-HIGGINS et STEWART (1964) qui ont d'abord démontré que la progression d'une vague vers le rivage dans la zone de surf provoque un excès de flux de la quantité de mouvement dans la direction de propagation. Ce forçage définit une composante en diagonale non-nulle si la vague est oblique au rivage. Ensuite, LONGUET-HIGGINS (1970) propose de calculer un courant moyen dans la zone de surf à partir de la direction et de la densité d'énergie de la houle au large (en acceptant l'application de la théorie linéaire de transformation de la houle). Dans cette approche, il est admis que le courant moyen dans la zone de surf varie uniquement longitudinalement: tout mouvement perpendiculaire au rivage est donc ignoré. Ce mouvement des masses d'eau en moyenne parallèle au rivage est couramment nommé dérive littorale depuis ces travaux. Le transport longitudinal désigne le déplacement des sédiments, lorsque ces derniers sont disponibles, par la dérive littorale (le courant). Plusieurs auteurs ont démontré l'importance de l'organisation spatiale du transport longitudinal sous formes de cellules, comme KOMAR et INMAN (1970) le long du littoral du sud de la Californie, ou plus tard comme BRAY et al. (1995) pour le sud de l'Angleterre. Il est couramment admis qu'une cellule de dérive littorale comprend une zone source de sédiments (érosion) 


\section{Thème 2 - Dynamique sédimentaire}

complétée par une zone puits (accumulation) adjacente, éventuellement séparées par une zone de transit; la dérive littorale permettant le transit des sédiments de la source vers le puits (KOMAR, 1976). Les cellules de dérive littorale sont aujourd'hui couramment utilisées comme cadre de gestion du rivage et leur détermination en Languedoc-Roussillon a fait l'objet de nombreuses études par le passé, synthétisées dans les thèses récentes de ALEMAN (2013), GERVAIS (2012) et BRUNEL (2010). Toutefois, l'organisation des cellules de cette région repose actuellement sur une connaissance empirique basée sur les variations du rivage, l'accumulation sédimentaire contre les digues portuaires, la granulométrie, des mesures courantologiques ponctuelles... mais une approche modélisatrice de la dérive littorale n’a jamais été entreprise à l'échelle régionale. De plus, les directions secondaires du transport longitudinal ne peuvent être connues selon ces méthodes. Cet article propose donc de combler ces lacunes par modélisation sur la base d'un climat de houle inédit et peu valorisé jusqu’à présent (ANEMOC-2).

\section{Site d'étude}

Les plages du Languedoc-Roussillon sont dominées par la houle et comportent généralement deux ou trois barres d'avant-côte. Ces plages pour lesquelles ALEMAN et al. (2015) ont affiné la typologie affichent une morphologie de type dissipatif à intermédiaire. Globalement, la taille des sédiments décroit du sud vers le nord, bien que des galets soient présents sur les plages des Aresquiers et du lido de Maguelone. La plupart des plages soufrent d'érosion chronique (figure 1B), et les transferts sableux longitudinaux définissent cinq grandes cellules de dérive littorale (DURAND, 1999 ; SABATIER, 2001 ; CERTAIN, 2002 ; MASSON et al., 2002 ; BRUNEL, 2010). Les houles morphogènes proviennent des secteurs $\mathrm{S}$ à $\mathrm{SE}$ dans le Languedoc tandis qu'elles prennent plutôt un cadran SSE à ESE dans le Roussillon, avec dans les deux cas des $\mathrm{H}_{1 / 3}$ annuelles proches de $4 \mathrm{~m}$. En outre, une rotation de la direction de ces houles est observée le long des plages du Languedoc-Roussillon (figure 1A) dans la donnée ANEMOC-2, ce qu'avait déjà relevé ALEMAN (2013) grâce aux mesures du réseau de bouées CANDHIS. Le marnage, qui est négligeable (environ $30 \mathrm{~cm}$ ), n'intervient pas sur l'organisation de la dérive littorale. 


\section{XIV vèmes Journées Nationales Génie Côtier - Génie Civil \\ Toulon, 29 juin au $1^{\text {er }}$ juillet 2016}
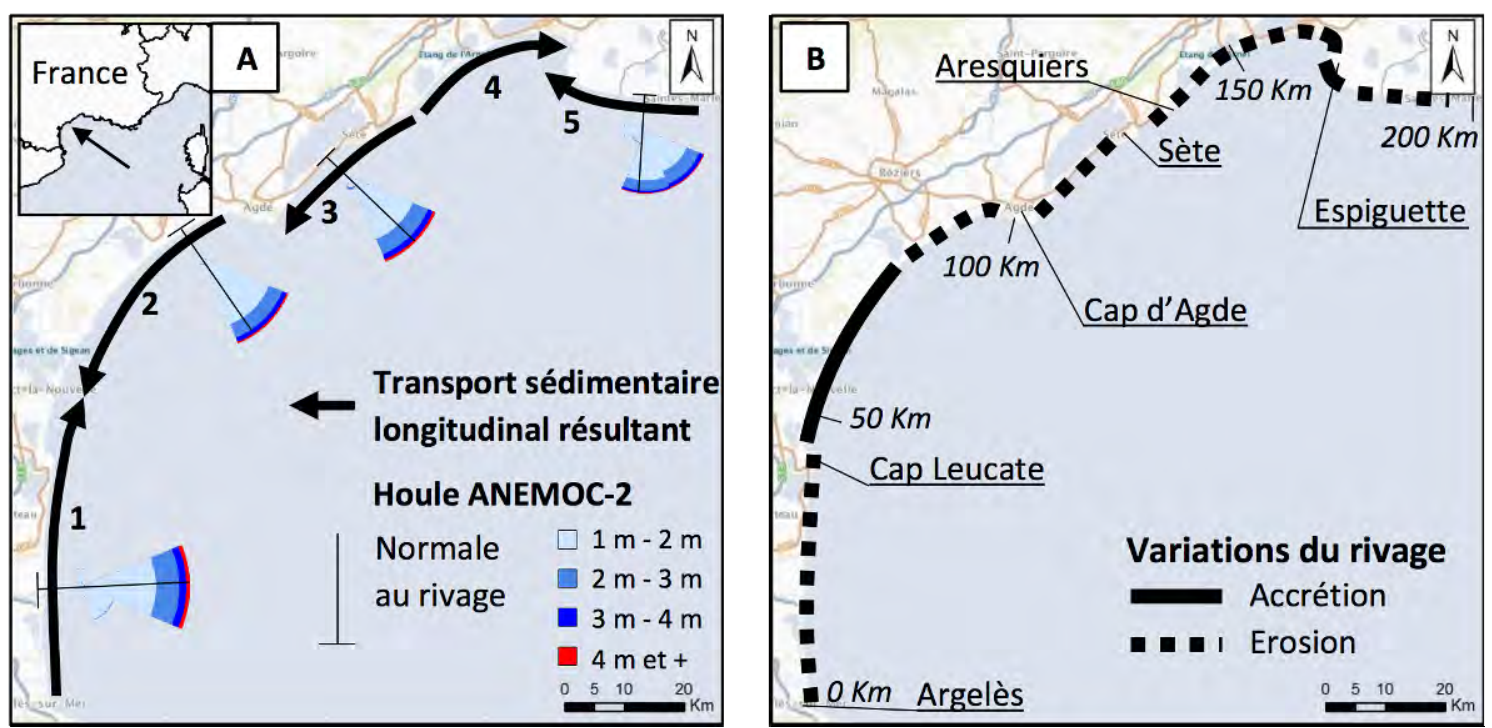

Figure 1. Cellules de dérive littorale et tendances évolutives du rivage en Languedoc-

Roussillon.

\section{Méthodologie}

\subsection{Rappels préliminaires}

La méthodologie pour estimer les variations du Transport Sédimentaire Longitudinal annuel Résultant $\left(\mathrm{TSL}_{\mathrm{R}}\right.$ ) reprend l'approche désormais classique décrite initialement par le SPM (USACE, 1984) dont nous rappelons les principes. Il s'agit de calculer le long du rivage (ici au moyen du modèle Unibest-LT) sur des profils topo-bathymétriques perpendiculaires à la côte, le transport sédimentaire annuel lié (exclusivement) à la dérive littorale. Le régime des houles variant en intensité mais aussi en direction, le transport se réalise donc alternativement vers la droite (TSL $_{D}$, volumes positifs par convention) et vers la gauche (TSL $\mathrm{T}_{\mathrm{G}}$, volumes négatifs par convention) des profils (observateur face au rivage). Si on admet un transport sédimentaire à l'équilibre, alors le $\mathrm{TSL}_{\mathrm{R}}$ de chaque profil est calculé par la différence (le Résultant) entre le TSL provenant de la droite et de la gauche (USACE, 1984). La qualité du calcul du TSL $L_{R}$ provient donc essentiellement des données en entrée au modèle (houle, bathymétrie) et de l'équation de transport sédimentaire utilisée. Cet article présente l'opportunité de valoriser des données en entrée récentes (LiDAR de 2011 et houles entre 1979-2010), et un modèle de transport longitudinal robuste (Unibest-LT).

\subsection{Procédure de calcul le long du rivage}

Afin que la propagation de la houle soit la plus précise possible, 116 points de sortie de modèle de houle issus de la base ANEMOC-2 sont utilisés en entrée des simulations Unibest-LT à une profondeur moyenne de $20 \mathrm{~m}$ (entre 15 et $40 \mathrm{~m}$ ). La base de données ANEMOC-2 est construite à partir de simulations issues du modèle spectral de $3^{\text {ème }}$ 


\section{Thème 2 - Dynamique sédimentaire}

génération TOMAWAC forcé avec des données de vent à $10 \mathrm{~m}$ de la NOAA (TIBERIWADIER et al., 2014). Elle offre des données horaires de hauteur, période et direction entre 1979 et 2010. La calibration repose sur une confrontation des sorties avec des données d'altimétrie spatiale et de bouées. Le spectre de houle est discrétisé en 36 directions et 32 fréquences $(0.0345$ à $0.66 \mathrm{~Hz}$, pour des périodes de $1,5 \mathrm{~s}$ à $29 \mathrm{~s})$. $\mathrm{Au}$ droit de chaque nœud ANEMOC-2 et perpendiculairement au rivage, un profil topobathymétrique est extrait des relevés LiDAR (2011). Sur ces profils, Unibest-LT applique l'approche recommandée par THORNTON et GUZA (1986) : la transformation de la houle du large vers le rivage par Unibest-LT repose sur le modèle probabiliste de BATTJES et JANSSEN (1978), tandis que le courant longitudinal est calculé selon le modèle de LONGUET-HIGGINS (1970). Le transport sédimentaire peut être simulé suivant différentes équations qui intègrent ou non les interactions vague-courant. Dans ce dernier cas, le calcul de la contrainte de cisaillement sur le fond rend compte de la combinaison non-linéaire de la friction sur le fond calculée pour la houle d'une part, et pour le courant longitudinal d'autre part. Les climats de houle ANEMOC-2 sont schématisés en classes de $25 \mathrm{~cm}$ pour la hauteur de houle et de $5^{\circ}$ pour les directions de provenance. Ce niveau de détail est à notre connaissance très rarement atteint dans la mise en œuvre d'Unibest-LT, d'autres auteurs ayant adopté une discrétisation plus grossière (RUGGIERO et al., 2010). Par conséquent, la répartition spatiale du calcul du TSL $\mathrm{T}_{\mathrm{R}}$ dépend directement de la distribution des nœuds de sorties de ANEMOC-2. Nos calculs présentent donc une résolution kilométrique, ce qui autorise une analyse à l'échelle de la région mais ne permet pas de décrire les circulations sédimentaires de manière plus détaillée (figure 2). A chaque profil topobathymétrique utilisé en entrée du modèle est associé la granulométrie de la zone de déferlement (figure 2) d'après les travaux de ALEMAN (2013) et SABATIER (2001).

\subsection{Calibration et tests de sensibilité}

Le modèle est testé et calibré sur la base d'une comparaison entre les volumes accumulés à la pointe de l'Espiguette entre 1984 et 2011 et ceux calculés numériquement. Les données de terrain témoignent d'une accumulation sédimentaire annuelle nette de $728 \mathrm{~K} \mathrm{~m}^{3}$ /an, dont $567 \mathrm{~K} \mathrm{~m}$ /an en amont-dérive de la digue de l'Espiguette et $161 \mathrm{~K} \mathrm{~m}$ /an en aval-dérive. Tout comme RUGGIERO et al. (2010), les tests de sensibilité et la calibration (SABATIER \& KULLING, 2015) indiquent que parmi les équations de transport proposées par Unibest-LT, celle de BIJKER (1971) procure les résultats les plus fiables, les volumes calculés par différentiel bathymétrique étant reproduits avec une marge d'erreur inférieure à $\pm 10 \%$ (les paramètres hydrauliques d’Unibest-LT sont utilisés avec leurs valeurs par défaut). Quant aux tests de sensibilité, ils montrent notamment une faible sensibilité de cette configuration à la granulométrie et à l'allure du profil de plage. Cependant, nous restons prudents sur 


\section{XIV èmes Journées Nationales Génie Côtier - Génie Civil \\ Toulon, 29 juin au $1^{\text {er }}$ juillet 2016}

l'interprétation des volumes calculés dans notre travail qui ne doivent être appréhendé que comme des ordres de grandeurs.

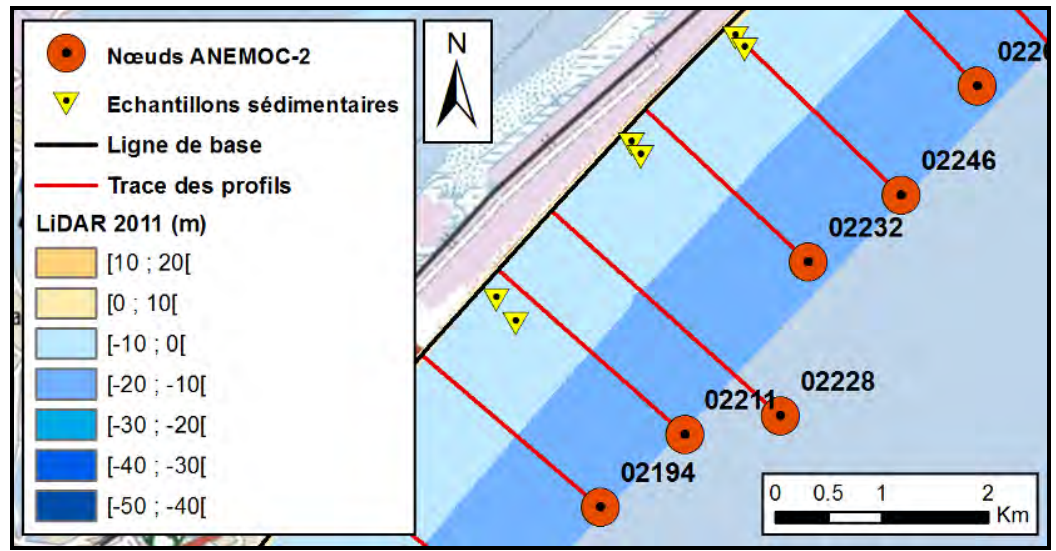

Figure 2. Exemple de nœuds ANEMOC-2 et axe des profils topo-bathymétriques.

\section{Résultats et discussion}

Dans l'ensemble du Languedoc-Roussillon, les directions du TSL $\mathrm{R}_{\mathrm{R}}$ (figures 3 et 4) correspondent généralement à celles issues des connaissances empiriques (figure 1A).

En Petite Camargue, le potentiel de $\mathrm{TSL}_{\mathrm{R}}$ est uniformément dirigé vers l'ouest (figure 3). Son intensité croît entre le Petit Rhône et le Rhône vif, passant de 600K $\mathrm{m}^{3} / \mathrm{an}$ à $700 \mathrm{~K} \mathrm{~m}$ /an environ et demeure stable jusqu'au phare de l'Espiguette. A partir de celui-ci, une décroissance du TSL $\mathrm{R}_{\mathrm{R}}$ est observée jusqu'à la digue de l'Espiguette où il se stabilise à $160 \mathrm{~K} \mathrm{~m}$ /an. Ces intensités sont en accord avec les travaux antérieurs (SABATIER, 2001) faisant état d'un transport dominant vers l'ouest de $861 \mathrm{~K} \mathrm{~m}$ /an $\pm 153 \mathrm{~K} \mathrm{~m}$ /an entre 1895 et 1974 mais aussi pour toutes les autres périodes d'observations. La direction du TSL $\mathrm{T}_{\mathrm{R}} \mathrm{s}$ explique par la proportion largement plus élevée du transport sédimentaire dirigé vers l'ouest car ce dernier est en moyenne 12 fois supérieur à celui dirigé vers l'est.

Entre Frontignan et Maguelone, le potentiel de TSL $\mathrm{R}_{\mathrm{R}}$ (350K $\mathrm{m}^{3}$ /an en moyenne) montre une divergence de direction au niveau des Aresquiers conformément aux connaissances empiriques (figures 1 et 3). A l'est des Aresquiers, l'organisation du TSL $\mathrm{TL}_{\mathrm{R}}$ est principalement causée par un transit dirigé vers le fond du golfe d'Aigues-Mortes (2 à 30 fois supérieur à celui de sens contraire). Alors qu'à l'inverse, à l'ouest de cette limite le transport dirigé vers Sète domine (jusqu'à 14 fois supérieur à celui orienté dans le sens contraire).

Le lido de Sète est caractérisé par un potentiel de TSL ${ }_{\mathrm{R}}$ dirigé vers le sud ne dépassant jamais les $200 \mathrm{~K} \mathrm{~m}^{3}$ /an (figure 3 ) : cette direction est identique à celle déterminée par les approches de géomorphologie expérimentale (figure 1). Cependant, le sens du TSL résulte d'une légère prédominance du transport dirigé vers le sud qui est en moyenne 2 fois supérieur à celui orienté vers le nord. Par conséquent, le lido de Sète doit connaître 


\section{Thème 2 - Dynamique sédimentaire}

un transport longitudinal bidirectionnel (surtout dans sa partie centrale), probablement en relation avec l'orientation générale du rivage et l'angle d'incidence des houles qui sont ici quasiment perpendiculaires au rivage, ce qui correspond théoriquement à une configuration peu propice à la dérive littorale.

Dans le Narbonnais, nos simulations indiquent une convergence du potentiel de TSL $L_{R}$ dans le secteur de Port la Nouvelle - grau de la Vieille Nouvelle (figure 4). Au nord de cette zone, le $\mathrm{TSL}_{\mathrm{R}}$ dirigé vers le sud montre un gradient décroissant entre Portiragnes $\left(600 \mathrm{~K} \mathrm{~m}^{3} / \mathrm{an}\right)$ et le grau de la Vieille Nouvelle ( $\left.50 \mathrm{~K} \mathrm{~m}^{3} / \mathrm{an}\right)$. Dans le détail, le transport dirigé vers le sud est 2 à 30 fois supérieur à celui dirigée vers le nord. Dans la zone de convergence, le $\mathrm{TSL}_{\mathrm{R}}$ devient bidirectionnel, avec des transits longitudinaux relativement équilibrés entre les deux directions. Au sud de la limite Port-la-Nouvelle -

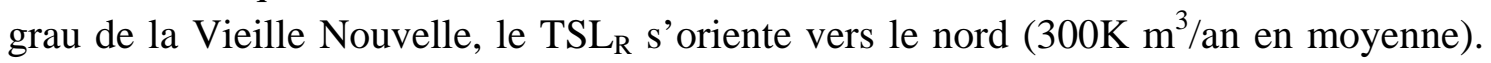
Cette direction du $\mathrm{TSL}_{\mathrm{R}}$ résulte d'un transit de direction nord en moyenne 5 fois supérieure à celle de sens opposé. Dans cette zone il est possible que notre stratégie de modélisation ne rende pas finement compte des processus de propagation de la houle au rivage. En effet, seul une modélisation 3D décrierait la zone d'ombre induite par le cap Leucate pour les houles de cadran sud (qui participent au transport sédimentaire vers le nord). Ici, le gradient longitudinal d'énergie des vagues doit décroitre de Port-LaNouvelle vers le Cap Leucate, ce qui peut éventuellement modifier localement la direction du transport longshore. En d'autres termes le calcul du TSL $L_{R}$ est probablement surestimé dans cette zone mais sa direction demeure satisfaisante, nos calculs concordant avec les connaissances empiriques.

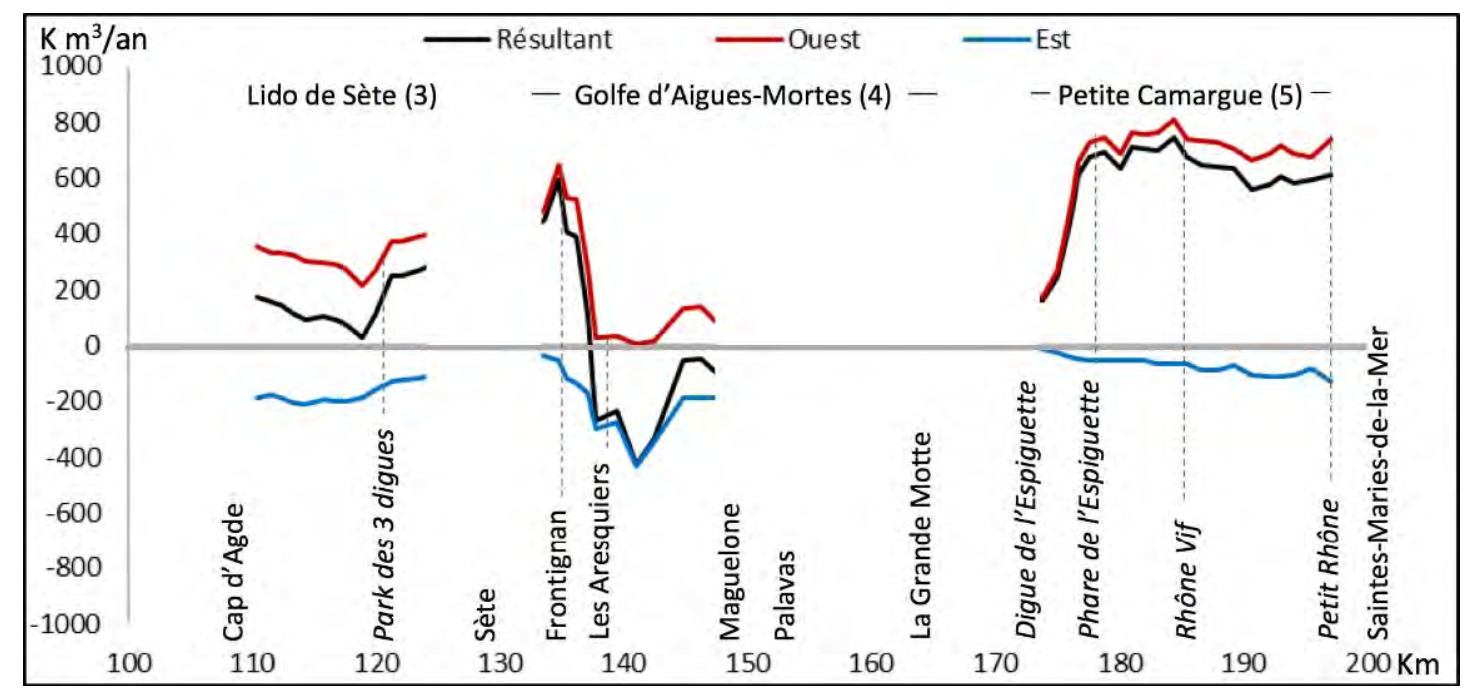

Figure 3. Potentiel de transport sédimentaire longitudinal résultant, vers l'est, vers l'ouest et total, du cap d'Agde au Petit Rhône. 


\section{XIV ${ }^{\text {èmes }}$ Journées Nationales Génie Côtier - Génie Civil \\ Toulon, 29 juin au $1^{\text {er }}$ juillet 2016}

Le potentiel de $\mathrm{TSL}_{\mathrm{R}}$ du Roussillon est globalement dirigé vers le nord (figure 4), d'une intensité moyenne de $300 \mathrm{~K} \mathrm{~m}$ /an mais pouvant atteindre $600 \mathrm{~K} \mathrm{~m}$ /an. Des inversions localisées sont présentes dans nos estimations mais celles-ci sont liées à la configuration particulière du rivage, contrôlée par des digues portuaires ou des morphologies d'embouchure fluviale. En conséquence, le transport dans la direction dominante chute drastiquement ponctuellement, tandis que celui de sens opposé augmente légèrement. Nos résultats montrent également qu'au nord de Port-Leucate, le TSL $_{R}$ devient sans doute bidirectionnel, les transits de directions contraires étant d’intensité équivalente.

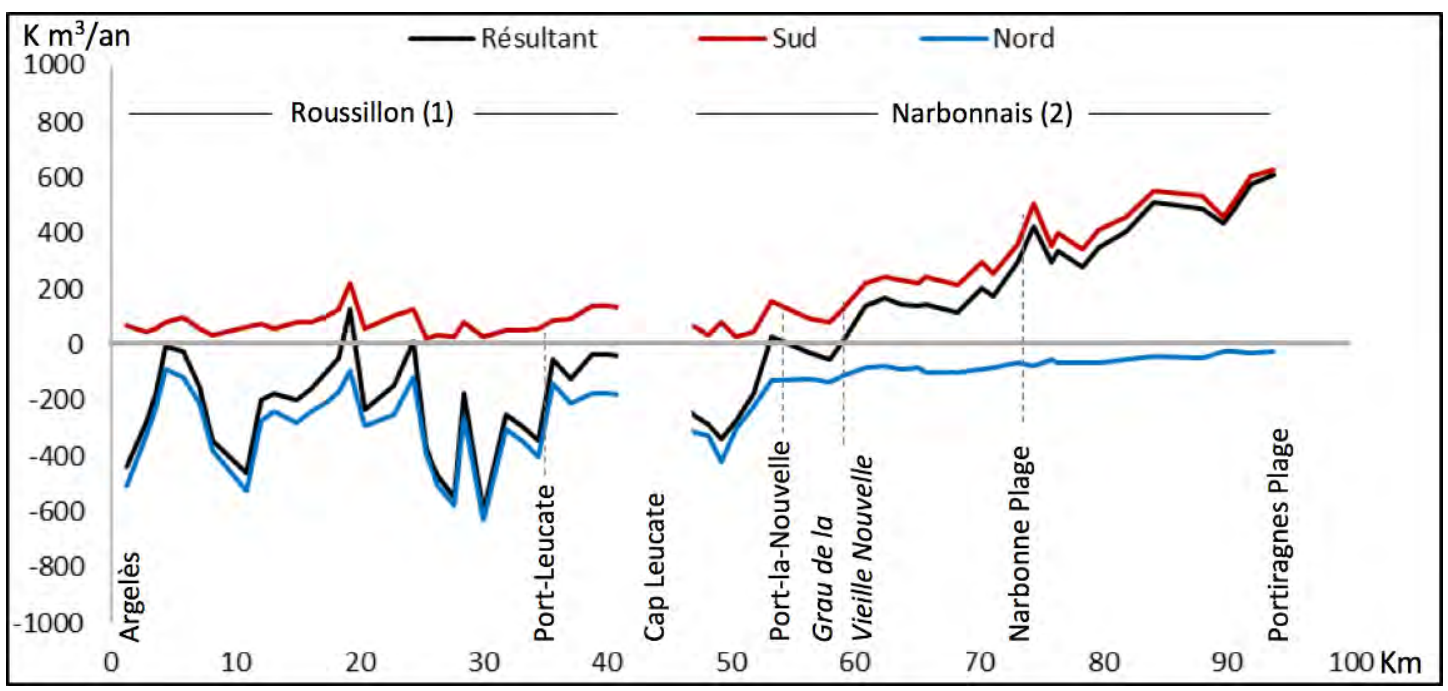

Figure 4. Potentiel de transport sédimentaire longitudinal résultant, vers le nord, vers le sud et total, d'Argelès à Portiragnes-Plage.

La comparaison des dérives littorales connues à des roses de houle ANEMOC-2 pour plusieurs positions dans le Languedoc-Roussillon (figure 1A) suggère que la rotation de la direction des houles le long des plages de la région est le moteur principal du transport longitudinal. Si globalement nos résultats montrent des potentiels de $\mathrm{TSL}_{\mathrm{R}}$ unidirectionnels, dans certains cas (lido de Sète, au nord de Port-Leucate) ils révèlent aussi des potentiels de transport longitudinal vers la droite et la gauche d'intensité relativement proche. En conséquence, un potentiel de $\mathrm{TSL}_{\mathrm{R}}$ a priori faible et unidirectionnel peut en réalité masquer des mouvements sédimentaires importants le long du rivage. En outre, à partir de nos estimations du transport longitudinal à l'échelle régionale, nous pouvons réaliser une première caractérisation des limites des cellules de dérive littorale. Ainsi, la digue de l'Espiguette constitue une limite bien localisée et fixe, mais poreuse, puisque nos résultats montrent un transport non nul au-delà de celle-ci. Tandis que dans le Narbonnais, la limite entre les dérives nord et sud est plus floue, située entre Port-la-Nouvelle et le grau de la Vieille Nouvelle. Le transport bidirectionnel au nord de Port-Leucate suggère une perturbation du transport entre le 


\section{Thème 2 - Dynamique sédimentaire}

Roussillon et le Narbonnais lorsque celui-ci est dirigé vers le sud. Néanmoins, l'analyse prochaine des variations interannuelles des potentiels de $\mathrm{TSL}_{\mathrm{R}}$ amènera à mieux caractériser la nature mobile ou fixe, poreuse ou perméable de ces limites.

\section{Conclusion}

La mise en œuvre d’Unibest-LT à l'échelle du littoral du Languedoc-Roussillon est satisfaisante à plus d’un titre. Les directions du TSL $\mathrm{T}_{\mathrm{R}}$ modélisé concordent bien avec celles définies empiriquement et nos travaux précisent et/ou dévoilent des dérives littorales potentielles unidirectionnelles ou bidirectionnelles selon les secteurs.

Par contre, il convient de garder à l'esprit le caractère conceptuel de l'interprétation du $\mathrm{TSL}_{\mathrm{R}}$ pour définir les cellules littorales car il s'agit d'une situation moyenne, dominante mais pour laquelle il est possible de trouver des différences d'intensités et/ou de directions selon les périodes d'observations. Par exemple, DURAND (1999) a montré, à partir de photographies aériennes, que la morphologie du grau de Gruissan suggère des inversions de dérives littorales ce qui s'oppose à nos résultats mais ne décrit pas une situation à moyen terme tel que le concept des cellules littorales le requiert.

De plus, la configuration d'Unibest-LT, validée le long de la pointe de l'Espiguette sur une côte aux houles régulièrement obliques et au transport unidirectionnel puis appliquée à l'ensemble des plages du Languedoc-Roussillon, où les conditions de dérives littorales sont moins optimales (incidence des houles au rivage plus faibles), induit peut-être un biais dans l'estimation du TSL ${ }_{R}$. Néanmoins, cette contrainte reste difficile à résoudre du fait de l'absence de mesures expérimentales fiables et utilisables pour calibrer et valider le modèle.

Ensuite, dans le Roussillon, la morphologie festonnée des barres d'avant côte autorise en théorie difficilement l'utilisation d'un modèle tel qu'Unibest-LT. Cependant, nos tests et les travaux de la littérature (cf. méthodologie) indiquent que la morphologie contrôle peu les résultats d'Unibest-LT lorsque l'équation de BIJKER (1971) est retenue, au contraire de l'incidence des houles au rivage. Par conséquent les résultats proposés par Unibest-LT restent acceptables dans le Roussillon. Toutefois, une analyse spatiale plus fine et/ou l'utilisation de modèles 3D produiraient logiquement des simulations plus précises. Par exemple, MICHAUD et al. (2012) proposent des résultats plus détaillés devant le littoral de Canet-Sainte-Marie au moyen d'un modèle 3D qui décrit plus finement les processus physiques qu'Unibest-LT. De plus, les modèles 3D présentent l'avantage d'aider à la description des processus responsables des dynamiques longitudinales et perpendiculaires au rivage, ces dernières étant bien identifiées dans le Languedoc-Roussillon (ALEMAN, 2013 ; GERVAIS, 2012). Les mouvements dans le profil ne sont pas décrits par Unibest-LT ni la modulation du courant longshore qui joue potentiellement un rôle sur les dynamiques perpendiculaires. Cependant, les modèles 3D demeurent encore très couteux en temps de calculs. Par exemple le travail de MICHAUD et al. (2012) concerne une zone de $2 \mathrm{~km}$ de long pour 


\section{XIV $V^{\text {èmes }}$ Journées Nationales Génie Côtier - Génie Civil \\ Toulon, 29 juin au $1^{\text {er }}$ juillet 2016}

simuler une tempête de 3 jours seulement, aussi notre démarche reste pertinente pour des zones géographiques plus vastes (ici près de $250 \mathrm{~km}$ ) surtout lorsque l'on recherche à établir des relations avec les cellules littorales dont la finalité consiste à apporter des bases solides aux gestionnaires du littoral. A l'avenir, les progrès numériques et informatiques autoriseront néanmoins une plus large intégration des processus physiques, y compris sur des domaines géographiques étendus et pour des durées affectant les processus à moyen terme (comme ceux qui contrôlent l'organisation d'un littoral en cellules). Dans cette perspective les mesures in situ demeurent toujours un préalable déterminant. Elles suggèrent par exemple la contribution des courants de vents depuis le plateau jusque dans les petits fonds (MICHAUD et al., 2012) et l'analyse des conditions de dérive littorale gagnerait donc aussi à être précisée vis-à-vis de ce forçage. Enfin, il est important de rappeler que les volumes proposés ici doivent être lus comme des ordres de grandeur correspondant à un potentiel de $\mathrm{TSL}_{\mathrm{R}}$.

\section{Remerciements}

Ce travail bénéficie d'un soutien financier (thèse) de la DREAL Languedoc-Roussillon initié par Bénédicte Guérinel que nous remercions ainsi que le relecteur anonyme pour ses remarques ayant contribué à améliorer la qualité de ce texte.

\section{Références bibliographiques}

ALEMAN N. (2013). Morphodynamique à l'échelle régionale d'une avant-côte microtidale à barres sédimentaires : le cas du Languedoc-Roussillon à l'aide de la technologie LiDAR. Thèse Université de Perpignan Via Domitia.

ALEMAN N., ROBIN N., CERTAIN R., ANTHONY E.J., BARUSSEAU J.P. (2015). Longshore variability of beach states and bar types in a microtidal, storm-influenced low-energy Mediterranean environment (Languedoc-Roussillon, France). Geomorphology, Vol. 241, pp 175-191. http://dx.doi.org/10.1016/j.geomorph.2015.03.029

BATTJES J.A., JANSSEN J.P.F.M. (1978). Energy loss and set-up due to breaking of random waves. Coastal Engineering Proceedings, pp 569-587. http://dx.doi.org/10.1061/9780872621909.034

BIJKER E.W. (1971). Longshore transport computations. Journal of the Waterways, Harbors and Coastal Engineering Division, Vol. 97(4), pp 687-703.

BRAY M.J., CARTER D.J., HOOKE J.M. (1995). Littoral Cell Definition and Budgets for Central Southern England. J. of Coastal Research, Vol. 11(2), pp 381-400.

BRUNEL C. (2010). Evolution séculaire de l'avant-côte de la méditerranée française, impact de l'élévation du niveau de la mer et des tempêtes. Thèse Aix-Marseille I.

CERTAIN R. (2002). Morphodynamique d'une cote sableuse microtidale à barres : le golfe du Lion (Languedoc-Roussillon). Thèse Université de Perpignan Via Domitia. 
DURAND P. (1999). L'évolution des plages de l'ouest du golfe du lion au XXème siècle. Cinématique du trait de côte, dynamique sédimentaire, analyse prévisionnelle. Thèse Université Lumière Lyon 2.

GERVAIS M. (2012). Impacts morphologiques des surcotes et vagues de tempêtes sur le littoral méditerranéen. Thèse Université de Perpignan Via Domitia.

KOMAR P.D. (1976). Beach Processes and Sedimentation. Prentice-Hall, Englewood Cliffs (New Jersey).

KOMAR P.D., INMAN D.L. (1970). Longshore Sand Transport on Beaches. Journal of Geophysical Research, Vol. 75(30), pp 5914-5927. http://dx.doi.org/10.1029/JC075i030p05914 LONGUET-HIGGINS M.S. (1970). Longshore currents generated by obliquely incident sea waves: 1. J. of Geophysical Research, Vol. 75(33), pp 6778-6789. http://dx.doi.org/10.1029/JC075i033p06778

LONGUET-HIGGINS M.S., STEWART R.W. (1964). Radiation stresses in water waves: A physical discussion, with applications. Deep Sea Research, Vol. 11, pp 529-562. http://dx.doi.org/10.1016/0011-7471(64)90001-4

MASSON M., CRAUCHET L., SABATIER F. (2002). Evolution du littoral sableux du Golfe du Lion. CETE Méditerranée, 85 p.

MICHAUD H., MARSALEIX P., LEREDDE Y., ESTOURNEL C., BOURRIN F., LYARD F., MAYET C., ARDHUIN F. (2012). Three-dimensional modelling of waveinduced current from the surf zone to the inner shelf. Ocean Science, Vol. 8(4), pp 657-681. http://dx.doi.org/10.5194/os-8-657-2012

RUGGIERO P., BUIJSMAN M., KAMINSKY G.M., GELFENBAUM G. (2010). Modeling the effects of wave climate and sediment supply variability on large-scale shoreline change. Marine Geology, Vol. 273, pp 127-140. http://dx.doi.org/10.1016/j.margeo.2010.02.008

SABATIER F. (2001). Fonctionnement et dynamiques morpho-sedimentaires du littoral du delta du Rhône. Thèse Université de Provence Aix-Marseille I.

SABATIER F., KULLING B. (2015) Modélisation du potentiel de transport longitudinal résultant annuel moyen sur l'avant-côte du Languedoc-Roussilon. Rapport Aix-Marseille Université pour la DREAL Languedoc-Roussillon, 84 p.

THORNTON E.B., GUZA R.T. (1986). Surf Zone Longshore Currents and Random Waves: Field Data and Models. American Meteorological Society, Vol. 16, pp 1165-1178. http://dx.doi.org/10.1175/1520-0485(1986)016<1165:szlcar>2.0.co;2

TIBERI-WADIER A.-L., LAUGEL A., BENOIT M. (2014). Construction of the numerical wave databases ANEMOC-2 on the Mediterranean Sea and the Atlantic Ocean through hindcast simulations over the period 1979-2010. SimHydro 2014, Sophia Antipolis.

USACE -U.S. Army Corps of Engineers- (1984). Shore Protection Manual, Coastal Engineering Research Center (U.S.), Washington, D.C. (2 volumes). 